\title{
Effect of Lumbar Spinal Stenosis on Bone Mineral Density in Osteoporosis Patients Treated With Ibandronate
}

Hyung-Youl Park

the Catholic University of Korea

Ji-Yoon $\mathrm{Ha}$

the Catholic University of Korea

Ki-Won Kim

the Catholic University of Korea

In-Hwa Baek

the Catholic University of Korea

Soo-Bin Park

the Catholic University of Korea

Jun-Seok Lee ( $\sim$ junband@naver.com )

the Catholic University of Korea

\section{Research Article}

Keywords: osteoporosis, lumbar spinal stenosis, ibandronate, bone mineral density

Posted Date: March 17th, 2021

DOI: https://doi.org/10.21203/rs.3.rs-289834/v1

License: (1) This work is licensed under a Creative Commons Attribution 4.0 International License. Read Full License 


\section{Abstract}

Background: Lumbar spinal stenosis (LSS) can cause various neurological symptoms and reduce the daily activity of patients. Many studies have shown that free physical activities and exercise can improve bone mineral density (BMD) in patients with osteoporosis. However, the effect of LSS on BMD has not been reported. The purpose of this study was to investigate the effects of LSS on BMD in patients treated with ibandronate for newly diagnosed osteoporosis.

Methods: Group 1 included 83 patients with osteoporosis alone, and group 2 included 76 patients with both osteoporosis and symptomatic LSS. We confirmed four BMD values presented as T-score at initial, and 1-, 2-, and 3-year follow-ups. Mean BMD and annual changes of BMD for three years were compared between the two groups. Correlations between initial BMD and total change of BMD were also analyzed in both groups.

Results: Mean annual BMDs were significantly higher in group 1 compared than in group 2 (-3.39 vs. -3.58 at 1 -year; -3.27 vs. -3.49 at 2 -year; -3.13 vs. -3.45 at 3 -year; all $p<0.05)$. Annual change of BMD at $1-$ year follow-up (0.32 vs. $0.21, p=0.036)$ and total change of BMD for three years $(0.57$ vs. $0.35, p=0.002)$ were significantly higher in group 1 . Group 1 had a strong negative correlation $(r=-0.511, P=0.000)$ between initial BMD and total change of BMD, whereas group 2 showed a weak negative correlation ( $r=$ $-0.247, p=0.032$ ).

Conclusions: Symptomatic LSS may interfere with BMD improvement in the treatment of osteoporosis with ibandronate. Active treatment for LSS with more potent treatment for osteoporosis should be taken to increase BMD for patients with osteoporosis and LSS.

\section{Background}

Osteoporosis is a systemic skeletal disorder in which bones become weak and vulnerable [1, 2]. Bisphosphonates are the potent bone resorption inhibitors. They include alendronate, risedronate, zoledronic acid, and ibandronate, which are widely used for the treatment of osteoporosis [3]. The action mechanism of bisphosphonate is that it can bind to an enzyme which acts in the pathway of osteoclast to inhibit the synthesis of cholesterol and decrease the formation of the proteins necessary for osteoclast function and survival, inducing osteoclast cell death [4]. Particularly, ibandronate can significantly reduce the risk of new vertebral fractures and increase bone mineral density (BMD) at the lumbar spine in postmenopausal women [5].

Lumbar spinal stenosis (LSS) is commonly associated with degenerative changes in which the spinal canal, lateral recess, and intervertebral foramen are narrowed and compress the cauda equina or nerve roots [6]. It can cause various neurological symptoms, such as lower back pain, intermittent claudication, and gait disturbance [7]. These neurological symptoms are known to reduce the daily activities of patients with LSS more than those of patients without LSS [8, 9]. Many studies have shown that physical activity and exercise can improve BMD in patients with osteoporosis [10-12]. 
The prevalence of osteoporosis is continuing to escalate with the increasing elderly population [1]. In the United States, the estimated prevalence was $10.3 \%$ (10.2 million) among adults aged 50 years or older in 2010 [13]. Lee et al. [14] have reported that $22.6 \%$ of postmenopausal patients with symptomatic LSS have osteoporosis, and $41.5 \%$ require treatment for osteoporosis in a cross-sectional study. As the prevalence of osteoporosis increases, studies on osteoporosis have also increased. However, to the best of our knowledge, the effect of LSS on BMD has not been reported. We hypothesized that LSS would have negative effect on BMD, because LSS causes neurological symptoms and eventually reduces the patient's physical activity. We aimed to evaluate the effect of LSS on BMD in patients undergoing osteoporosis treatment with ibandronate.

\section{Materials And Methods Study population}

From January 2004 to December 2017, we retrospectively reviewed 398 patients in a single institution who had been initially diagnosed with postmenopausal osteoporosis and who had started taking osteoporosis medications. Clinical parameters, including age, sex, body mass index (BMI), and BMD, were assessed by means of patients' electronic medical records.

This study was approved by the Institutional Review Board (IRB) in accordance with the Declaration of Helsinki (approval number.

PC18RESE0034).

The informed consents were waived by IRB (The Catholic University of Korea, Eunpyeong St. Mary's Hospital) because of the retrospective study design.

Inclusion criteria were: (1) undergoing BMD evaluation every year for three years after the initial BMD test in patients with newly diagnosed osteoporosis (a total of four BMD evaluations), and (2) $150 \mathrm{mg}$ oncemonthly oral ibandronate treatment for three years. All patients received calcium (600 $\mathrm{mg} / \mathrm{day})$ and vitamin D (400 international units/day) as an adjunctive therapy.

Exclusion criteria were: (1) taking steroid hormones, (2) surgery or fracture of spine or lower extremities, (3) malignant tumor or medical conditions that reduced daily activities, (4) rheumatoid arthritis, (5) metabolic bone disease, and (6) gait disorders due to causes other than LSS. Finally, 159 patients were included in this study.

\section{Diagnosis of symptomatic LSS}

Symptomatic LSS were defined as obvious central stenosis on magnetic resonance imaging (MRI) and clinically related neurological symptoms. According to MRI-based classification system for central stenosis by Lee et al. [15], patients with grade 2 or 3 central stenosis were included. Patients with concomitant foraminal stenosis or lateral recess stenosis, combined with central stenosis, were also included $[16,17]$. Clinically related neurological symptoms were as following: (1) pain, weakness or 
numbness in the legs, calves or buttocks when standing or walking, (2) neurological claudication; cramping in the calves with walking, requiring frequent short rests to walk a distance, and (3) symptoms that improve when sitting or bending forward [6].

We divided the patients into two groups according to the presence or absence of symptomatic LSS at the time of diagnosis for osteoporosis. Patients who did not complain of LSS symptoms were included as group 1 (osteoporosis group), while group 2 included patients with both osteoporosis and symptomatic LSS (osteoporosis + spinal stenosis group) (Fig. 1). All patients with LSS were treated conservatively with medications. Patients who underwent surgery for worsening of stenosis during the follow-up period were excluded from this study.

\section{Measurement of BMD}

Osteoporosis was confirmed by dual-energy X-ray absorptiometry (Lunar Prodigy; GE Healthcare BioSciences Corp., Piscataway, NJ, USA) for all patients. A BMD T-score of -2.5 or less in the total lumbar spine was defined as osteoporosis [18].

We confirmed a total of four BMD values presented as T-score, initial and at 1-, 2-, and 3-year follow-ups, in each patient at the total lumbar spine or same vertebral bodies where the initial BMD was assessed.

We obtained annual changes of BMD in each group by calculating the difference in mean BMDs for two consecutive years (annual change of BMD = BMD in index year $-B M D$ in the previous year).

We assessed a total change of BMD for three years by calculating the difference between initial mean $B M D$ and mean BMD at three years later (a total change of BMD = BMD on 3-year follow-up - initial $B M D$ ). To investigate the improvement of osteoporosis according to the initial BMD in each group, we assessed correlations between initial BMD and total change of BMD in both groups.

\section{Statistical analyses}

We compared means of continuous variables using a paired $t$-test within each group and unpaired Student's $t$-test between the groups. The correlation between initial BMD and total change of BMD was analyzed by the Pearson correlation coefficient, and simple linear regression was simultaneously conducted. All statistical analyses were done using the SPSS software (IBM SPSS Statistics, Version 24.0, IBM Corp., Armonk, NY, USA). Values for $p<0.05$ were considered significant.

\section{Results}

Table 1 shows patient characteristics between the two groups. The number of patients was 83 in group 1 , and 76 in group 2. There were no significant differences in mean age, BMI, and initial BMD between the two groups. 
Table 1

Patient characteristics between the two groups.

\begin{tabular}{|llll|}
\hline & $\begin{array}{l}\text { Group 1 } \\
\text { (osteoporosis) }\end{array}$ & $\begin{array}{l}\text { Group 2 } \\
\text { (osteoporosis + LSS) }\end{array}$ & P-value \\
\hline Patients number & 83 & 76 & \\
\hline Female : male & $83: 0$ & $76: 0$ & 1.000 \\
\hline Age (years) & $73.7 \pm 9.6$ & $74.8 \pm 8.9$ & 0.492 \\
\hline Height (cm) & $154.2 \pm 8.0$ & $152.1 \pm 4.8$ & 0.284 \\
\hline Weight (kg) & $56.3 \pm 9.8$ & $53.6 \pm 8.5$ & 0.352 \\
\hline BMI (kg/m²) & $23.6 \pm 3.3$ & $23.1 \pm 3.0$ & 0.609 \\
\hline Initial BMD (T-score) & $-3.71 \pm 0.49$ & $-3.80 \pm 0.77$ & 0.388 \\
\hline LSS, lumbar spinal stenosis; BMI, body mass index; BMD, bone mineral density \\
\hline
\end{tabular}

\section{Comparison within the group}

In group 1, mean BMDs improved significantly every year during the three-year follow-up (-3.71 \pm 0.49 vs. $-3.39 \pm 0.42$ vs. $-3.27 \pm 0.48$ vs. $-3.13 \pm 0.45$, all $p<0.001$ ) (Fig. 2 ). In group 2 , mean BMD at 1 -year followup improved significantly compared to the initial BMD $(-3.80 \pm 0.77$ vs. $-3.58 \pm 0.76, p<0.001)$. However, mean BMDs at 2-and 3-year follow-ups did not improve significantly compared to the previous year $(-3.58 \pm 0.76$ vs. $-3.49 \pm 0.75, p=0.064 ;-3.49 \pm 0.75$ vs. $-3.45 \pm 0.80, p=0.424)$ (Fig. 2$)$.

\section{Comparison between the two groups}

Table 2 shows the mean BMDs and annual changes of BMDs during follow-up. Mean BMDs at annual follow-up for three years were significantly higher in group 1 than in group 2. The annual change of BMD at the 1-year follow-up was significantly higher in group 1 than in group 2. However, there were no differences in the annual change of BMD at the 2-year and 3-year follow-ups between the two groups. A total change of BMD for three years was also significantly higher in group 1 than in group 2. 
Table 2

$\mathrm{BMD}$, annual change of $\mathrm{BMD}$, and a total change of $\mathrm{BMD}$ in the two groups.

\begin{tabular}{|llll|}
\hline & $\begin{array}{l}\text { Group 1 } \\
\text { (osteoporosis) }\end{array}$ & $\begin{array}{l}\text { Group 2 } \\
\text { (osteoporosis + LSS) }\end{array}$ & P-value \\
\hline BMD & & & \\
\hline Initial & $-3.71 \pm 0.49$ & $-3.80 \pm 0.77$ & 0.388 \\
\hline at 1-year F/U & $-3.39 \pm 0.42$ & $-3.58 \pm 0.76$ & 0.045 \\
\hline at 2-year F/U & $-3.27 \pm 0.48$ & $-3.49 \pm 0.75$ & 0.034 \\
\hline at 3-year F/U & $-3.13 \pm 0.45$ & $-3.45 \pm 0.80$ & 0.003 \\
\hline Annual change of BMD & & & \\
\hline at 1-year F/U & $0.32 \pm 0.29$ & $0.21 \pm 0.34$ & 0.036 \\
\hline at 2-year F/U & $0.12 \pm 0.28$ & $0.09 \pm 0.44$ & 0.702 \\
\hline at 3-year F/U & $0.14 \pm 0.25$ & $0.04 \pm 0.41$ & 0.069 \\
\hline Total change of BMD & $0.57 \pm 0.41$ & $0.35 \pm 0.50$ & 0.002 \\
\hline LSS, lumbar spinal stenosis; BMD, bone mineral density; F/U, follow-up \\
\hline ation between initial BMD and total Change of BMD in
\end{tabular}

Group 1 showed a strong negative correlation between initial BMD and total change of BMD $(r=-0.511, p$ $=0.000 ;$ Fig. 3A). Group 2 showed a weak negative correlation between initial BMD and total change of $\operatorname{BMD}(r=-0.247, p=0.032 ;$ Fig. 3B).

\section{Discussion}

We found that ibandronate increased BMDs in patients newly diagnosed with osteoporosis. In both groups, the mean BMD at the 3-year follow-up improved significantly compared to the initial value (-3.71 vs. -3.13 for group $1 ;-3.80$ vs. -3.45 for group 2 , all $p<0.001)$. This result was consistent with a previous report showing that BMDs at the lumbar spine and hip increased significantly (3.1\% and $1.8 \%$, respectively; $p<0.0001$ vs. placebo) with daily 2.5 -mg ibandronate after 24 months [19].

In patients with osteoporosis alone, the annual BMD increased significantly over the previous BMD for three years, which suggests that the therapeutic effect of ibandronate is significant every year in patients with osteoporosis alone. However, in patients with osteoporosis and LSS, the annual BMD increased significantly from the initial value only during the first year but did not show a significant increase after that, which suggests that in patients with osteoporosis and LSS, the efficacy of ibandronate was sufficient for the first year, after which the effect of the treatment decreased, perhaps because of the 
progression of spinal stenosis over time, worsening the symptoms of stenosis, and thus reducing the physical activities of patients, which may reduce the therapeutic effect of ibandronate.

LSS causes neurologic claudication and reduces the strength of the lower limb, which decreases physical activity [10]. Walking difficulty due to claudication or physical inactivity can be associated with decreased BMD [20]. A previous study reported a relationship between physical inactivity and decreased BMD in patients with vascular claudication originating from peripheral arterial disease [20]. Physical activities in seniors can induce the maintenance of BMD or increase BMD by means of physical load [11, 21, 22]. Lee et al. [23] have also reported that increased physical activity and regular walking exercise could prevent osteoporosis in a study of older women aged 65 years and over. In the present study, the direct comparison of annual BMDs between the two groups over three years showed that the annual BMDs in patients with osteoporosis and LSS were significantly lower than in patients with osteoporosis alone, which suggests that LSS may interfere with the improvement of BMD in the treatment of osteoporosis.

These findings were consistent with the correlations between initial BMD and total change of BMD in both groups. The correlation analysis showed that in patients with osteoporosis alone, the initial BMD and the total change of BMD had a strong negative correlation. It suggests that in patients with osteoporosis alone, the more severe the initial osteoporosis, the higher the degree of osteoporosis improvement after treatment. However, in patients with osteoporosis and LSS, there was a weak negative correlation between initial BMD and total change of BMD, which means that the relationship between the severity of initial osteoporosis and the degree of osteoporosis improvement after treatment is weaker in patients with osteoporosis and LSS than in those with osteoporosis alone.

In patients with LSS, a decrease in the therapeutic effect of ibandronate may also be associated with deterioration of bone metabolism [24]. Lee et al. [14] reported that $55.6 \%$ of patients with LSS had hypovitaminosis $D$, which reduced the effectiveness of osteoporosis treatment. Kim et al. [25] reported that limited physical activity in symptomatic LSS patients resulted in high bone turnover rates, including bone formation and bone resorption markers. Moreover, in a subsequent study, they reported that decompression surgery for symptomatic LSS patients had a positive effect on bone metabolism by reducing the increased bone resorption rate [26]. These reports show that improved walking ability and physical activity resulting from the active treatment of LSS can help improve the effectiveness of osteoporosis treatment. Therefore, active treatment for LSS to alleviate neurological symptoms, combined with more potent treatment for osteoporosis should be taken to increase BMD for patients with osteoporosis and LSS.

This study has some limitations. First, we did not make an objective measure that daily activity was lower in patients with osteoporosis and LSS than in patients with osteoporosis alone. Previous studies have reported that daily activity decreases in patients with LSS, so we were able to conduct this study based on the earlier results [8-10]. Further research using objective tools, such as questionnaires on daily activities, is needed. Second, we could not include bone turnover markers in this study. Because it was retrospective, test values were not present for some patients. Third, this study included only patients 
treated with ibandronate. Thus, the results of this study may not apply to patients treated with other types of osteoporosis drugs. Additional prospective trials are needed to validate our findings and extend these results to different kinds of osteoporosis drugs. Finally, clinical outcomes of osteoporosis treatment, such as osteoporotic fractures, were not evaluated during the follow-up, because we focused only on whether LSS affects BMD in the treatment of osteoporosis. Despite these limitations, our study has the strength that it is the first case series to evaluate the effect of LSS on BMD in the treatment of osteoporosis patients.

\section{Conclusions}

We have demonstrated that BMD increased less in patients with osteoporosis and LSS than in patients with osteoporosis alone during osteoporosis treatment with ibandronate. This result supported that symptomatic LSS may interfere with BMD improvement in the treatment of osteoporosis patients. Therefore, active treatment for LSS to increase the physical activities, combined with more potent treatment for osteoporosis should be taken to increase BMD for patients with osteoporosis and LSS.

\section{Abbreviations}

BMD, bone mineral density; LSS, lumbar spinal stenosis; BMI, body mass index; IRB, Institutional Review Board; MRI, magnetic resonance imaging

\section{Declarations}

\section{Acknowledgments}

None

\section{Funding}

This work was supported by a Small Grant for Exploratory Research (SGER) through the Ministry of Education of the Republic of Korea and The Catholic University of Korea Songeui (2018R1D1A1A02049202).

\section{Availability of data and materials}

The datasets generated and/or analyzed during the current study are available from the corresponding author on reasonable request.

\section{Authors' contributions}

JSL conceived, designed, and led the study. JYH, SBP and IHB prepared the figures and collected the data. PHY and JSL analyzed the data and wrote the manuscript. KWK made contributions to reviewing the manuscript. All authors read and approved the final manuscript. 


\section{Competing interests}

All authors declare that they have no competing interests.

\section{Consent for publication}

Not applicable.

\section{Ethics approval and consent to participate}

The data were recorded and analyzed anonymously. The study protocol was reviewed and approved by the Institutional Review Board of the Catholic University of Korea (PC18RESE0034). The informed consents were waived by the IRB because of the retrospective study design.

\section{Author details}

${ }^{1}$ Department of Orthopedic Surgery, Eunpyeong St. Mary's Hospital, College of Medicine, the Catholic University of Korea, Seoul, Korea. ${ }^{2}$ Department of Orthopedic Surgery, Yeouido St. Mary's Hospital, College of Medicine, the Catholic University of Korea, Seoul, Korea.

\section{References}

1. Tu KN, Lie JD, Wan CKV, Cameron M, Austel AG, Nguyen JK, et al. Osteoporosis: A Review of Treatment Options. P t. 2018;43:92-104.

2. Tokeshi S, Eguchi Y, Suzuki M, Yamanaka H, Tamai H, Orita S, et al. Relationship between Skeletal Muscle Mass, Bone Mineral Density, and Trabecular Bone Score in Osteoporotic Vertebral Compression Fractures. Asian Spine J. 2020.

3. Zhou J, Ma X, Wang T, Zhai S. Comparative efficacy of bisphosphonates in short-term fracture prevention for primary osteoporosis: a systematic review with network meta-analyses. Osteoporos Int. 2016;27:3289-300.

4. Rizzoli R. Bisphosphonates for post-menopausal osteoporosis: are they all the same? Qjm. 2011;104:281-300.

5. Chesnut CH, 3rd, Skag A, Christiansen C, Recker R, Stakkestad JA, Hoiseth A, et al. Effects of oral ibandronate administered daily or intermittently on fracture risk in postmenopausal osteoporosis. $\mathrm{J}$ Bone Miner Res. 2004;19:1241-9.

6. de Schepper El, Overdevest GM, Suri P, Peul WC, Oei EH, Koes BW, et al. Diagnosis of lumbar spinal stenosis: an updated systematic review of the accuracy of diagnostic tests. Spine (Phila Pa 1976). 2013;38:E469-81.

7. Shamji MF, Mroz T, Hsu W, Chutkan N. Management of Degenerative Lumbar Spinal Stenosis in the Elderly. Neurosurgery. 2015;77 Suppl 4:S68-74.

8. Martinelli TA, Wiesel SW. Epidemiology of spinal stenosis. Instr Course Lect. 1992;41:179-81. 
9. Johnsson KE. Lumbar spinal stenosis. A retrospective study of 163 cases in southern Sweden. Acta Orthop Scand. 1995;66:403-5.

10. Kirkaldy-Willis WH, Paine KW, Cauchoix J, Mclvor G. Lumbar spinal stenosis. Clin Orthop Relat Res. 1974:30-50.

11. Honda A, Sogo N, Nagasawa S, Shimizu T, Umemura Y. High-impact exercise strengthens bone in osteopenic ovariectomized rats with the same outcome as Sham rats. J Appl Physiol (1985). 2003;95:1032-7.

12. Chastin SF, Mandrichenko O, Helbostadt JL, Skelton DA. Associations between objectively-measured sedentary behaviour and physical activity with bone mineral density in adults and older adults, the NHANES study. Bone. 2014;64:254-62.

13. Wright NC, Looker AC, Saag KG, Curtis JR, Delzell ES, Randall S, et al. The recent prevalence of osteoporosis and low bone mass in the United States based on bone mineral density at the femoral neck or lumbar spine. J Bone Miner Res. 2014;29:2520-6.

14. Lee BH, Moon SH, Kim HJ, Lee HM, Kim TH. Osteoporotic profiles in elderly patients with symptomatic lumbar spinal canal stenosis. Indian J Orthop. 2012;46:279-84.

15. Lee GY, Lee JW, Choi HS, Oh KJ, Kang HS. A new grading system of lumbar central canal stenosis on MRI: an easy and reliable method. Skeletal Radiol. 2011;40:1033-9.

16. Wildermuth S, Zanetti M, Duewell S, Schmid MR, Romanowski B, Benini A, et al. Lumbar spine: quantitative and qualitative assessment of positional (upright flexion and extension) MR imaging and myelography. Radiology. 1998;207:391-8.

17. Steurer J, Roner S, Gnannt R, Hodler J. Quantitative radiologic criteria for the diagnosis of lumbar spinal stenosis: a systematic literature review. BMC Musculoskelet Disord. 2011;12:175.

18. Siris ES, Adler R, Bilezikian J, Bolognese M, Dawson-Hughes B, Favus MJ, et al. The clinical diagnosis of osteoporosis: a position statement from the National Bone Health Alliance Working Group. Osteoporos Int. 2014;25:1439-43.

19. McClung MR, Wasnich RD, Recker R, Cauley JA, Chesnut CH, 3rd, Ensrud KE, et al. Oral daily ibandronate prevents bone loss in early postmenopausal women without osteoporosis. J Bone Miner Res. 2004;19:11-8.

20. Fahrleitner-Pammer A, Obernosterer A, Pilger E, Dobnig H, Dimai HP, Leb G, et al. Hypovitaminosis D, impaired bone turnover and low bone mass are common in patients with peripheral arterial disease. Osteoporos Int. 2005;16:319-24.

21. Anand A, Shetty AP, Renjith KR, K SS, Kanna RM, Rajasekaran S. Does Sarcopenia Increase the Risk for Fresh Vertebral Fragility Fractures?: A Case-Control Study. Asian Spine J. 2020;14:17-24.

22. Shah GM, Gong HS, Chae YJ, Kim YS, Kim J, Baek GH. Evaluation and Management of Osteoporosis and Sarcopenia in Patients with Distal Radius Fractures. Clin Orthop Surg. 2020;12:9-21.

23. Lee I, Ha C, Kang $\mathrm{H}$. Association of sarcopenia and physical activity with femur bone mineral density in elderly women. J Exerc Nutrition Biochem. 2016;20:23-8. 
24. Thakkar P, Prakash NB, Tharion G, Shetty S, Paul TV, Bondu J, et al. Evaluating Bone Loss with Bone Turnover Markers Following Acute Spinal Cord Injury. Asian Spine J. 2020;14:97-105.

25. Kim HJ, Lee HM, Kim HS, Park JO, Moon ES, Park H, et al. Bone metabolism in postmenopausal women with lumbar spinal stenosis: analysis of bone mineral density and bone turnover markers. Spine (Phila Pa 1976). 2008;33:2435-9.

26. Kim HJ, Lee HM, Chun HJ, Kang KT, Kim HS, Park JO, et al. Restoration of bone turnover rate after decompression surgery in patients with symptomatic lumbar spinal stenosis: preliminary report. Spine (Phila Pa 1976). 2009;34:E635-9.

\section{Figures}




\section{Patients initially diagnosed with osteoporosis $(n=398)$}

from January 2004 to December 2017

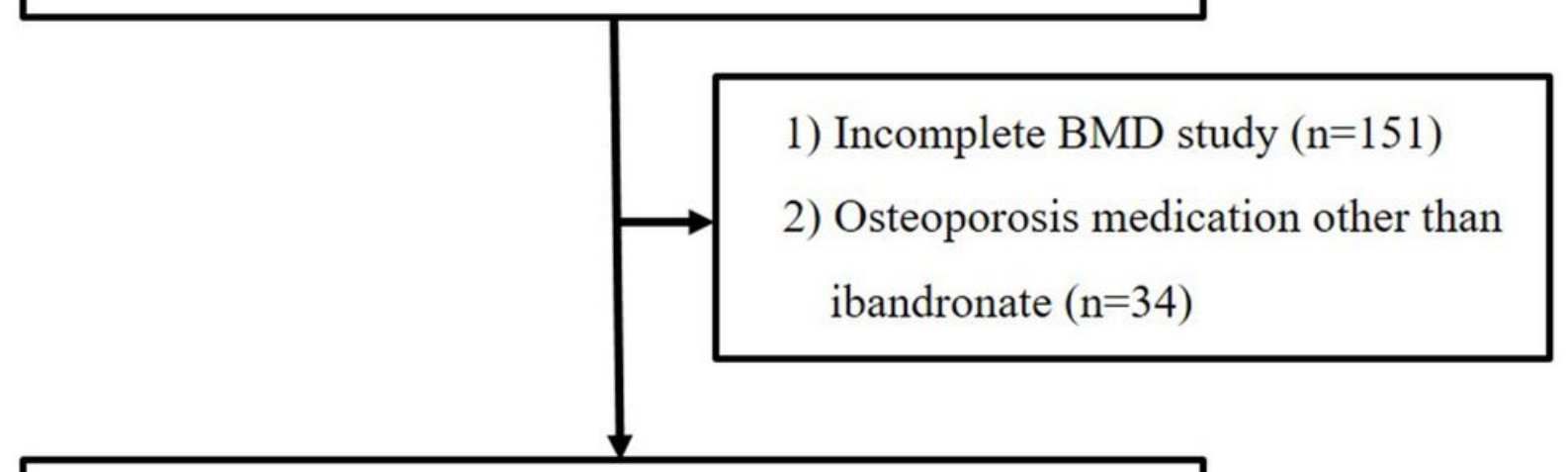

Patients satisfying criteria for inclusion $(n=213)$

\section{Exclusion}

1) Intake of steroid hormones $(n=7)$

2) Surgery or fracture of spine or hip $(n=35)$

3) Malignant tumor or severe medical conditions $(n=9)$

4) Gait disturbance without spinal stenosis $(n=3)$

\section{Total patients included in the study $(n=159)$}

Group 1 (osteoporosis, $\mathrm{n}=83$ )

Group 2 (osteoporosis + spinal stenosis, $\mathrm{n}=76$ )

\section{Figure 1}

Flow chart showing the inclusion of patients in this study. 


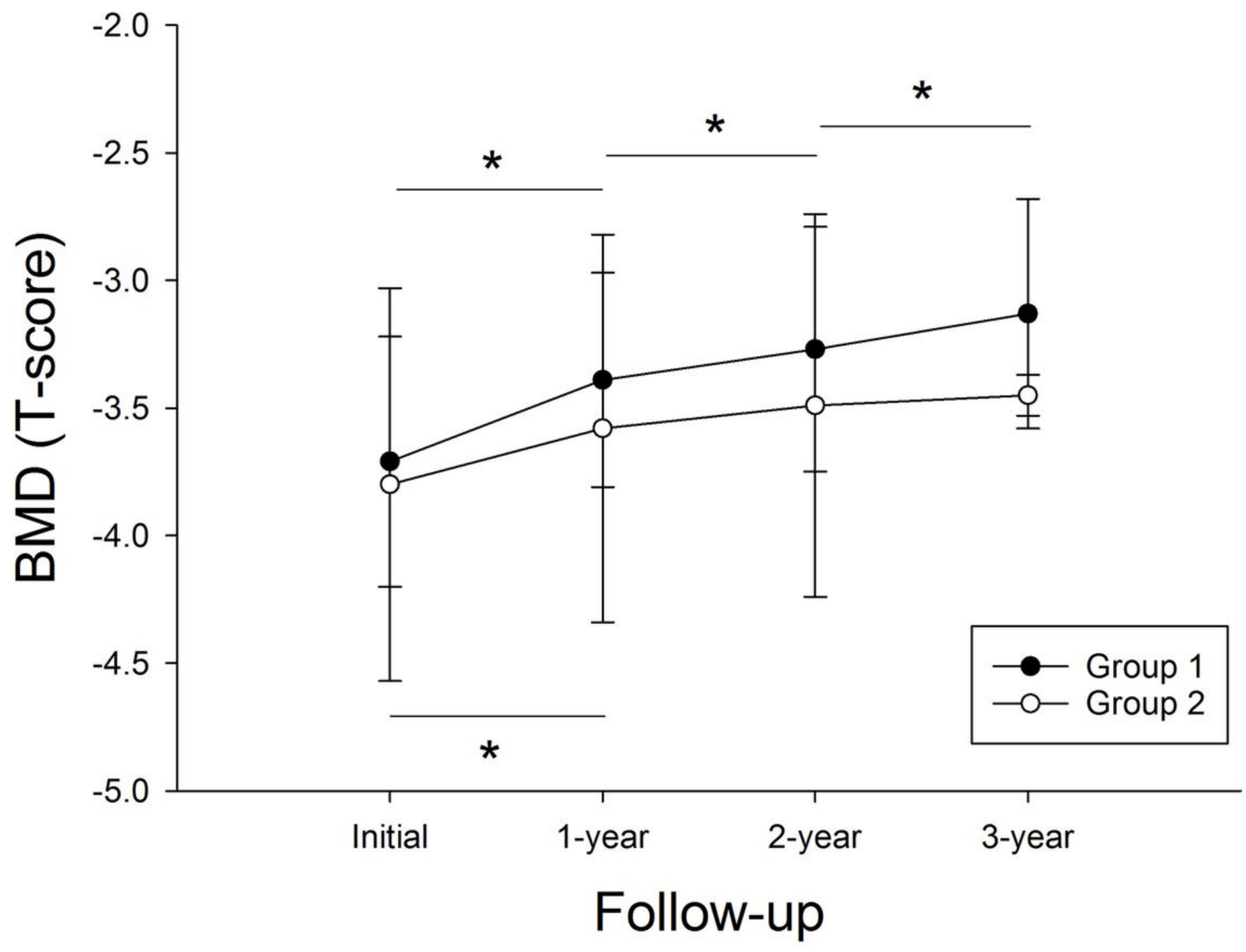

Figure 2

Annual BMDs in the two groups. * indicates $p<0.05$. 

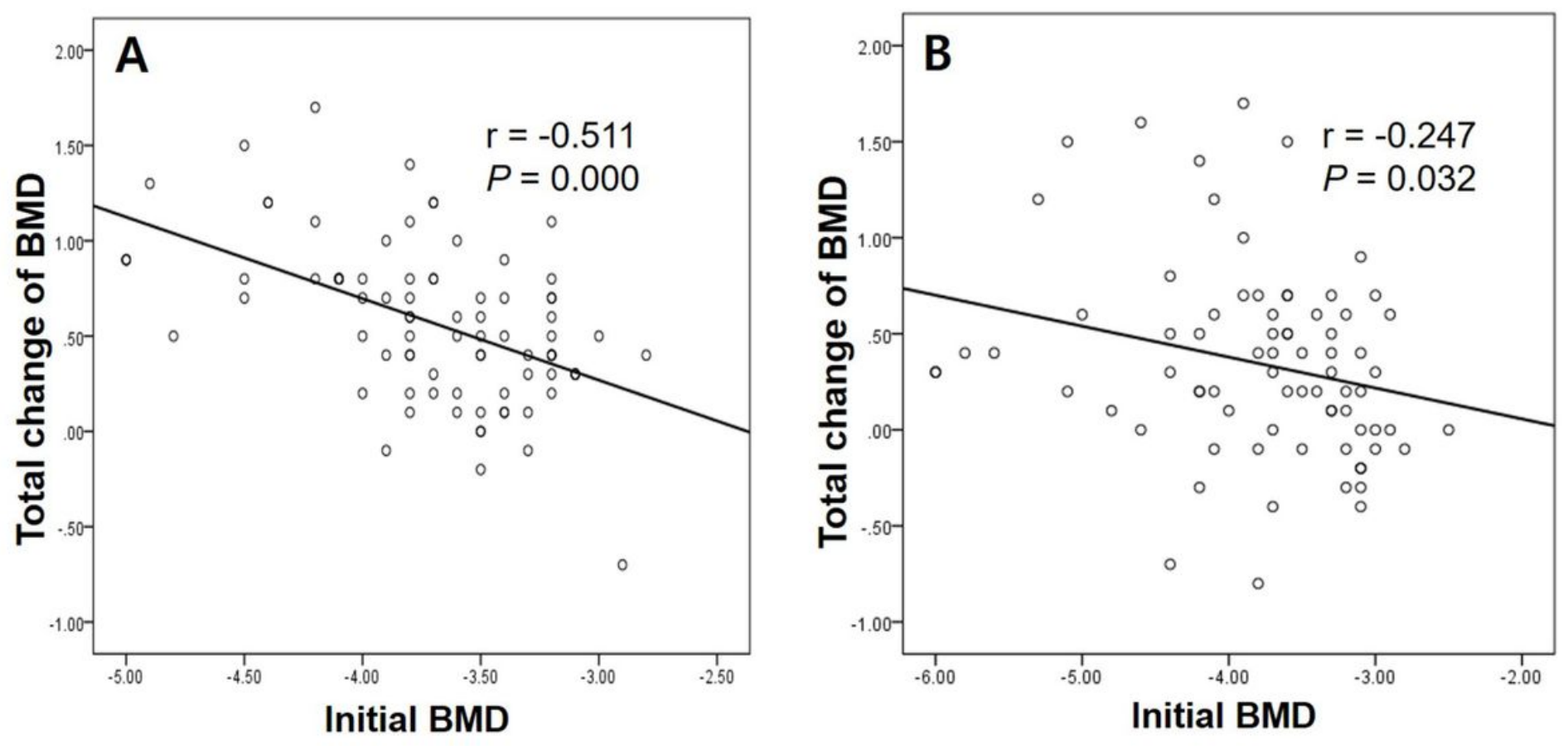

Figure 3

Correlations between initial BMD and total change of BMD in (A) group 1 and (B) group 2. 